\title{
Report on the 4th International Conference on Autonomous Infrastructures, Management, and Security (AIMS 2010) and the International Summer School on Network and Service Management (ISSNSM 2010)
}

\author{
Stiller, Burkhard ; De Turck, Filip ; Morariu, Cristian ; Waldburger, Martin
}

\begin{abstract}
This article contains the report on AIMS 2010, which was held June 23-25, 2010 at the Department of Informatics (IFI), University of Zürich, Switzerland and which was driven by the theme "Mechanisms for Autonomous Management of Networks and Services". Furthermore, the report also synopsizes ISSNSM 2010, which was run June 21-23, 2010 at the same location and which offered a practical summer school on selected topics in network and service management.
\end{abstract}

DOI: https://doi.org/10.1007/s10922-010-9190-9

Posted at the Zurich Open Repository and Archive, University of Zurich

ZORA URL: https://doi.org/10.5167/uzh-55731

Journal Article

Accepted Version

Originally published at:

Stiller, Burkhard; De Turck, Filip; Morariu, Cristian; Waldburger, Martin (2011). Report on the 4th International Conference on Autonomous Infrastructures, Management, and Security (AIMS 2010) and the International Summer School on Network and Service Management (ISSNSM 2010). Journal of Network and Systems Management, 19(1):130-136.

DOI: https://doi.org/10.1007/s10922-010-9190-9 


\title{
Report on the 4th International Conference on Autonomous Infrastructures, Management, and Security (AIMS 2010) and the International Summer School on Network and Service Management (ISSNSM 2010)
}

\author{
Burkhard Stiller ${ }^{1}$, Filip De Turck ${ }^{2}$, Cristian Morariu ${ }^{1}$, Martin Waldburger ${ }^{1}$ \\ ${ }^{1}$ University of Zürich, CSG@IFI, Binzmühlestrasse 14, CH-8050 Zürich, Switzerland \\ [stiller|morariu|'waldburger]@ifi.uzh.ch \\ ${ }^{2}$ Ghent University-IBBT, Gaston Crommenlaan 8, B-9050 Gent, Belgium \\ filip.deturck@intec.ugent.be
}

\begin{abstract}
This article contains the report on AIMS 2010, which was held June 23-25, 2010 at the Department of Informatics (IFI), University of Zürich, Switzerland, driven by the theme "Mechanisms for Autonomous Management of Networks and Services". Furthermore, the report presents ISSNSM 2010, which was run June 21-23, 2010 at the same location, offering a practical summer school on selected topics in network and service management.
\end{abstract}

Keywords: Network Management, Service Management, Autonomous Infrastructures, Security

\section{INTRODUCTION}

The 4th International Conference on Autonomous Infrastructures, Management, and Security (AIMS 2010) and the 4th International Summer School on Network and Service Management (ISSNSM'08) was held in a collocated manner for 2.5 days each at the University of Zurich, Switzerland, on June 21-25, 2010 [1], [2]. The entire technical and local organization had been performed by the Communication Systems Group (CSG) of the Department of Informatics (IFI) at the University of Zurich, which was supported technically and financially by the European FP6 Network of Excellence (NoE) for the "Management of Internet Technologies and Complex Services" (EMANICS) [3], seeing further support from the FP7 Specific Targeted REsearch Project (STREP) SmoothIT (Simple Economic Management Approaches of Overlay Traffic in Heterogeneous Internet Topologies) [4]. Additionally, AIMS 2010 was co-sponsored by IFIP TC6.6 [5] and the CSG@IFI [6].

This report summarizes the core concept followed in AIMS and ISSNMS for the fourth time. It shows an approach with good benefits for students and interested researchers in a field of highly interrelated theoretical and practical aspects. Therefore, this report discusses briefly the technical program of AIMS and ISSNSM. The 5th AIMS and ISSNSM are already planned for 2011 and potentially interested people will be welcome to check for details, following the new links to be established originating from [1].

\section{CONCEPT AND APPROACH}

The 2.5-day AIMS conference [1] was planned and run as a single-track event. Preceded by the summer school, the AIMS program itself included besides regular paper sessions, a key note, and a tutorial Ph.D. workshop sessions. This approach allows for an exchange of ideas in research between Ph.D. students and senior researchers. The theme of AIMS 2010 was "Mechanisms for Autonomous Management Networks and Services" and focussed - besides naming a paper session on autonomous management — on mechanisms in support of decentralized management approaches.

Summer schools have found a quite important position in teaching and education by today, especially addressed to students and researchers, who are interested to get attached to a new field of expertise in a limited period of time. Thus, EMANICS determined that the stringent demand to educate Ph.D. students in the area of network and service management across affiliations and country borders. After the 1st ISSNSM hosted by the Jacobs University Bremen, Germany, the 2nd ISSNSM hosted by the University of Zürich, Switzerland, already, and the 3rd ISSNSM hosted by University of Federal Armed Forces Munich, Germany, the 4th ISSNSM returned to Zürich. It was clearly focused on extended practical parts, which means that in about one third of the time, typical classroom teaching took place and in the other two thirds practical lab courses had been organized and run, which addressed the respective topic's key aspects.

Thus, the full understanding of theoretical concepts and practical approaches, tools, and systems has proven to be a highly successful summer school concept, since those lab courses and experiments prepared deepened the knowledge of the topic extremely well. The work in practice, at an example, and with the systems at hands - fully supervised by the respective instructor(s) - included the application of knowledge, which typically a pure one-way lecture or a book reading cannot provide.

\section{AIMS KEYNOTE AND TUTORIAL}

The past of AIMS shows that industrial key notes are very welcome to address real life problem areas as well as constraints of less academic, but business-driven type. In that context the key note presentation was given by Metin Feridun, IBM Research Zurich, Switzerland, on "Facing Complexity in Systems Management". While Metin has worked in management-related topics for years this talk outlined that 
emerging technologies, such as cloud computing, increased the use, proliferation, and mobility of powerful end-user devices. In addition, the migration of applications to the Internet as of today are creating exciting challenges to the management of IT (Information Technology) infrastructures in general. Those include amongst others massive scale and distribution of IT resources, the expectation of high availability of Internet-based services, and a heterogeneity of resources, all of which increase the complexity system administrators encounter. Therefore, optimized approaches are needed for these problems, which cover service management as well as configuration management aspects in an ever increasing software and system basis such that "old" components need to be continued in reliable operation and "new" ones have to be integrated at the same time seamlessly.

The AIMS tradition to offer fully integrated tutorials - thus no need to register or pay more - has been continued again. Therefore, Luca Deri, from ntop, Italy, an expert in accounting management, has been invited to talk about "Large-scale Flow Monitoring Through Open Source Software". He argued that large and high-speed networks produce a large number of flows that need to be collected and analyzed. However, since collectors are unable to keep up with the flow export rate and also have severe speed limitations when creating reports, this tutorial provides insights into an appropriate solution. Due to recent innovation in databases, combined with existing opensource software applications, a very well suited flow collection and exploration of large-scale flows becomes feasible. This is extended by the Web 2.0 technology to enable network administrators to analyze data collected in real-time and explore them interactively by means of a Web browser.

\section{AIMS PROGRAM AND TOPICS}

The technical paper sessions included topics in the areas of "P2P-based Systems", "Autonomous Management", and "Management Mechanisms", which have been complemented by a "Short Paper Session" on various interesting topics of emerging, but still on-going work. All appeared in the proceedings [7]. Based on 27 submissions the three technical paper sessions have been filled with 9 papers selected. Additionally, 5 short papers have been selected, too. For each full paper a 20 minutes time slot for the presentation had been assigned, followed by 10 minutes space for questions and discussions. Thus, such timing leaves an optimal amount of time, where without any pressure of next presentation time slots or sessions, a tremendous amount of time has been used successfully.

To be able to value the best paper within the AIMS conferences, a best paper award committee had been established, including Marinos Charalambides (UCL, U.K.), Ramin Sadre (University of Twente, The Netherlands), and the two TPC co-chairs of AIMS, namely Filip De Turck (Ghent University, Belgium) and Burkhard Stiller (University of Zürich, Switzerland). Based on the best formal review results three papers have been closely analyzed and their presentation had been taken into consideration. Finally, two papers have received the split Best Paper Award - financially sponsored by IFIP TC6.6: Shaonan Wang, Radu State, Mohamed Ourdane, Thomas Engel: "Mining NetFlow Records for Critical Network Activities" and Kaloyan Kanev, Nikolay Melnikov, Jürgen Schönwälder: "Implementation of a Streambased IP Flow Record Query Language". While the former paper investigates an intelligent data-mining technique for discovering relevant information in huge network monitoring datasets, the latter paper describes in detail an undertaken implementation of a flow query language, which overcomes existing limitations in complexity and performance. For their scientific and technical detail, refer to the full papers at [7].

\section{AIMS PH.D. WORKSHOP}

Such a Ph.D. workshop approach and format is quite unique and was integrated into the AIMS program as in previous years to show that Ph.D. research in its initial stage or upon finalizations gives raise to discussions, views, and suggestions. Thus, those 11 presentations of Ph.D. projects, selected out of 22 proposals originally submitted, had to present in 10 minutes each their core idea, the solution approach, and the challenges faced. All Ph.D. workshop papers haven been organized into two sessions on "Overlays and Non-conventional Network Infrastructures" and "Security, Network Monitoring, and Analysis".

Questions posed in these two sessions covered aspects like "What is the relevance of your problem identified?", "In which way do you see an application of your work in tomorrows IT systems?", and "Why shall someone apply your expected results in an operational manner?". The discussion of advantages and drawbacks continued during the breaks and did establish new human connections of expertise.

\section{Summer School Program AND Topics}

The 2.5-day summer school [2] had selected 3 topics out of a number of proposals collected from within the EMANICS community and beyond. Those 3 topics covered the areas of (1) Wireless Sensor Network Management, (2) Device Management, and (3) Traffic Mining.

\section{A. A Practical Introduction to 6LoWPAN: Programming IPv6 Wireless Sensor Networks with Contiki}

Today, wireless networks consisting of low powered devices find an increasing number of application areas in fields like vehicular networks, sensor networks, mobile ad-hoc networks, and smart grids. Low power networks are used in different ways, such as to retrieve sensor data or to manage and control devices. Hence, the need for interoperability has emerged.

The development of protocols, which are suited for low power embedded devices is challenging in dimensions of highly limited computing power, low data rates, and energy constraints. Accordingly, the 6LoWPAN standard has been developed to address these challenges accrediting as it provides a method for low power embedded devices to communicate using IPv6 over IEEE 802.15.4 networks.

Consequently, the aim of this tutorial was to introduce attendees to programming embedded devices. To that aim, virtual machines running the Contiki OS (Operating System) 
were used for setting up IPv6-enabled wireless sensor networks. Attendees were also introduced to the 6LoWPAN standard. In practical exercises attendees learned the basics of programming with Contiki and were walked through setting up an IPv6 network using (real) sensor network motes. Attendees gained experience in developing their own custom applications by learning how to send and receive messages via the UDP protocol over the IPv6 network.

\section{B. Embedded Automation Systems and Device Manageability Instrumentation}

The operation of network-based IT applications and services in a professional and timely manner has become an increasingly challenging task over the last few years. New challenges embrace, for example, (a) the ongoing virtualization and distributed deployment of business-critical applications and services or (b) an increase in bandwidth requirements, network links, and number of transactions per seconds on those links. This situation is complemented by different operational dimensions of contractual SLA (Service Level Agreement) obligations, legal requirements, industry standards, and by dynamically changing user and application expectations.

Accordingly, this tutorial has shown attendees in which ways concepts of self-* and autonomic systems have been finding their way from research and academia into the networking industry, and how manageability and embedded automation capabilities with the network itself have evolved. In particular, attendees were explained how network elements today differ from what they were a few years ago as they now offer a plethora of embedded functionality in order to measure, detect, decide, and act upon information from within the network.

This tutorial made use of a combination of theory and practical hands-on examples to discuss the capabilities of Embedded Automation Systems. This included a detailed look at implications, benefits, and challenges of using embedded automation. Technology examples used covered Cisco's IOS $₫$ embedded manageability instrumentation, such as IP SLA, Embedded Event Manager (EEM), Flexible NetFlow (FNF), Network Based Application Recognition (NBAR) and Embedded Packet Capture (EPC).

\section{Traffic Mining: Feel the Packets, be the Packets}

The IP network introduced a new quality of threat, when real infrastructures, such as telecommunication, electrical, transport and banking systems, were infiltrated by the virtual world with its standard PC hardware and software. Software became an end in itself and a weapon. Unnecessary high complexity, unstable behavior, and a new kind of criminal activity arose, the targeted IT warfare and the ignorance of the administrative management toward technical and human complexity issues. Nevertheless, this situation was accepted and promoted regardless of the fact that this was violating the most basic principles of reliable design, testing, and robustness. The general acceptance of product selection solemnly by economic and political factors demands now its toll.

This tutorial, thus, addressed reasons for today's calamity and it motivated the current civil and military research efforts to solve the most urgent problems on a short and long time scale. The tutorial embraced practical insight into the practice of infrastructure troubleshooting and traffic mining with respect to the danger of today's trust in tools instead of decent human knowledge. Attendees, hence, were taught the potential of the human brain, mining in IP data streams using KISS (Keep It Small and Simple) tools on selected examples, such as Skype, but also on real world processed datasets in legal agreement with the owner. The positive magic of critical thinking, proper preprocessing, and visually exploring of datasets have been demonstrated. Besides practical considerations also the dark side of the mining was discussed shortly. All what you should not do includes: The application of Churchill's law, the negative magic of statistical spin doctors, an insufficient preprocessing, balancing heuristics versus facts, "wish you were here" or "get there effect", the tool-fool syndrome.

\section{EXPERIENCES AND NEXT STEPS}

This year's summer school 2010 was attended by 15 persons from 7 different countries, which originated from 10 different affiliations. Furthermore, 5 persons attending ISSNSMS did not belong to EMANICS, thus, showing that the interest in network and service management runs well beyond the NoE's limits. The majority of attendees came from universities representing Ph.D. students. In addition, the number of registered participants for AIMS was 41, who originated from 14 different countries and from which about $50 \%$ have neither been EMANICS nor SmoothIT project members.

The goal of a highly interactive, discussion-open, and relaxed environment had been achieved, since interactions between Ph.D. students themselves as well as senior researchers had happened. Even the technical excursion to the Technorama in Winterthur (Swiss Science Center) [8], providing hands-on experiments in the fields of mechanics, magnetism, electricity, perception, math-magics, and water/ nature/chaos, followed by a joint dinner has attracted almost all of those attendees registered to join and enjoy!

Due to very positive feedback for AIMS and ISSNSM received and those stimulating discussions observed, the conference and the summer school for 2011 is in the planning phase and will continue as the 5 th event with a similar concept as described.

\section{REFERENCES}

[1] AIMS 2010 Web Site: http://www.aims-conference.org/2010/

[2] ISSNSM 2010 Web Site, http://www.aims-conference.org/issnsm-2010/

[3] EMANICS Project Web Site, http://www.emanics.org/, July 2010.

[4] SmoothIT Project Wen Site: http://www.smoothit.org/, July 2010.

[5] IFIP TC6 Working Group 6: "Management of Networks and Distributed Systems", http://www.simpleweb.org/ifip/, July 2010.

[6] Communication Systems Group CSG, Department of Informatics IFI, University of Zürich UZH: http://www.csg.uzh.ch/, July 2010.

[7] B. Stiller, F. De Turck (Edts.): Mechanisms for Autonomous Management of Networks and Services, Proceedings of AIMS 2010, Lecture Notes in Computer Science LNCS Vol. 6155, Springer Verlag, Heidelberg, Germany, 2010.

[8] Technorama - The Swiss Science Center: http://www.technorama.ch/, July 2010. 


\section{A. Author Biographies}

Prof. Dr. Burkhard Stiller chairs as a full professor the Communication Systems Group CSG, Department of Informatics IFI at the University of Zürich UZH since 2004. He holds a Computer Science Diplom and a Ph.D. degree of the University of Karlsruhe, Germany. During his research locations of the Computer Laboratory, University of Cambridge, U.K., the Computer Engineering and Networks Laboratory, ETH Zürich, Switzerland, and the University of Federal Armed Forces, Munich, Germany. His main research interests cover, including current CSG topics, charging and accounting of Internet services, economic management, systems with a fully decentralized control (P2P), telecommunication economics, and biometric management systems. He participates in a number of European, industrial, and Swiss research projects and serves as a technical program committee member as well as chair of several conferences.

Prof. Dr. Filip De Turck is a full-time professor since 2006 affiliated with the Department of Information Technology of the Ghent University and the IBBT (Interdisciplinary Institute of Broadband Technology Flanders) in the area of telecommunication and software engineering. He holds a Ph.D. degree in Electronic Engineering from Ghent University. His main research interests include scalable software architectures for telecommunication network and service management, performance evaluation and design of new telecommunication and eHealth services. He participates in various research projects with industry and academia on these topics and serves as program committee member of several conferences and workshops in this research area.

Cristian Morariu received his Masters of Science degree from Technical University of Cluj-Napoca, Romania in June 2004. His major at the Faculty of Automation and Computer Science, was performed in Computer Science. While holding an ERASMUS scholarship he developed his Master Thesis at the Swiss Federal Institute of Technology (ETH Zürich), Laboratory of Software Technology. Since September 2004 he is a doctoral student at the University of Zurich, Department of Informatics, Communication Systems Group. His main interests are in the area of IP accounting and distributed architectures for traffic analysis.

Martin Waldburger Martin Waldburger holds a Master of Science (M.Sc.) degree, which he received in 2004 from the University of Zürich. In the same year, he joined Prof. Dr. Burkhard Stiller's Communication Systems Group (CSG) at the University of Zurich in the position of an assistant and doctoral student. He participated in the European Union project "Access to Knowledge through the Grid in a Mobile World" (Akogrimo) and the "European Union Network of Excellence for the Management of Internet Technologies and Complex Services" (EMANICS). His research work is concerned with technical, legal, and business aspects of electronic service provisioning in multi-domain environments. In particular, he is focusing on research challenges in automated contract formation for value-added electronic services in an international context. 